\title{
Rethinking the role of epinephrine in cardiac arrest: the PARAMEDIC2 trial
}

\author{
Julianna Jung, Julie Rice, Sharon Bord \\ Johns Hopkins University School of Medicine, Baltimore, MD, USA \\ Correspondence to: Julianna Jung. Johns Hopkins University School of Medicine, Baltimore, MD, USA. Email: jjung@jhmi.edu. \\ Provenance: This is an invited Editorial commissioned by Section Editor Rongkai Yan, MD (Division of Biostatistics and Bioinformatics, Department \\ of Oncology, University of Johns Hopkins University, Baltimore, USA). \\ Comment on: Perkins GD, Ji C, Deakin CD, et al. A Randomized Trial of Epinephrine in Out-of-Hospital Cardiac Arrest. N Engl J Med \\ 2018;379:711-21.
}

Submitted Nov 30, 2018. Accepted for publication Dec 14, 2018.

doi: 10.21037/atm.2018.12.31

View this article at: http://dx.doi.org/10.21037/atm.2018.12.31

Epinephrine (adrenaline) is the most commonly used medication in cardiac arrest, and is the only drug recommended by the American Heart Association (AHA) for all arrests regardless of cardiac rhythm (1). The use of epinephrine in cardiac arrest originated from studies in the 1960's, when it was found to improve survival in dog models of asphyxia (2). Physiologically, epinephrine produces some effects that may be helpful and others that may be harmful. It is an agonist at both beta- and alphaadrenergic receptors. The alpha effects cause peripheral vasoconstriction, which in turn leads to increased blood flow in the central circulation, improving perfusion of the heart and brain. These effects are potentially beneficial, and have been hypothesized to increase the likelihood of return of spontaneous circulation (ROSC) among cardiac arrest patients. The beta-adrenergic effects increase cardiac rate, contractility, and automaticity; these effects are potentially deleterious, leading to arrhythmias and increased myocardial oxygen demand.

The use of epinephrine in cardiac arrest has never been supported by rigorous scientific data. However, the role of epinephrine in cardiac arrest has only begun to be questioned in recent years. In 2009, a Norwegian study found that patients in cardiac arrest who were treated with basic life support interventions (CPR and defibrillation only) had identical outcomes to those treated with advanced cardiac life support interventions (basic life support plus parenteral medications) (3). A post hoc analysis from this study specifically examined the role of epinephrine, finding evidence of improved short-term survival among patients who received it. However these patients had decreased long-term survival rates and worse neurologic outcomes (4). These findings cast doubt on the role of "advanced" interventions, and raise questions about whether the longstanding practice of administering epinephrine to patients in cardiac arrest is justified.

Most studies of epinephrine have been observational in nature. In one study of out-of-hospital arrest patients, epinephrine was associated with a significant increase in the likelihood of ROSC, but it was also associated with a decrease in the likelihood of 30-day survival or favorable neurologic outcome (5). In another study focusing specifically on arrest patients who achieved ROSC, outcomes were compared between patients who received epinephrine and those who did not. This study found significantly lower rates of neurologically intact survival among patients receiving epinephrine, and a dosedependent effect was observed, wherein higher doses of epinephrine were associated with lower odds of favorable outcome (6).

One group in Australia undertook a randomized placebocontrolled trial of epinephrine in out-of-hospital cardiac arrest patients. In this study, 601 patients were randomized, and the authors found increased rates of ROSC and survival to hospital admission in the epinephrine group. Although the epinephrine group showed a trend towards increased rates of survival to hospital discharge, it did not achieve statistical significance. Study authors attributed the inability to demonstrate longer-term benefit to difficulties with patient recruitment. Because it withheld a "standard of 
care" medication from critically ill cardiac arrest patients, the study received negative attention from politicians and the press. This resulted in the loss of study sites and participants, and ultimately only one of the five ambulance services in the region chose to participate (4).

Lin et al. conducted a systematic review and metaanalysis of studies on epinephrine in cardiac arrest, and found a mere 14 articles-a surprisingly small number given that epinephrine has been considered "standard of care" for decades. Of these studies, there was only one placebocontrolled trial (the Australian trial described above). Other studies compared standard- to high-dose epinephrine, and standard-dose epinephrine to vasopressin with or without epinephrine. The meta-analysis supported prior findings that administration of epinephrine was associated with higher likelihood of ROSC, but that there was no evidence of benefit in terms of survival to hospital discharge or favorable neurologic outcome (7).

The lack of rigorous experimental studies on the clinical outcomes associated with epinephrine has led the resuscitation community to continue to recommend its use in cardiac arrest. However, the PARAMEDIC2 trial might be a true game-changer in how clinicians think about epinephrine. The study, conducted by Perkins et al. in the United Kingdom, included 8,014 patients with outof-hospital cardiac arrest. Patients were randomized to receive either epinephrine $(n=4,015)$ or placebo $(n=3,999)$. Primary outcome was 30 -day survival, and secondary outcomes included survival to hospital discharge and neurologically intact status. The authors found that administration of epinephrine increased 30-day survival rates $3.2 \%$ in the epinephrine group, compared to $2.4 \%$ in the placebo group). However, a larger proportion of patients in the epinephrine group were neurologically devastated, with modified Rankin scores of $4-5$ (31\% in the epinephrine group, compared to $17.8 \%$ in the placebo group). This result explains the lack of overall improvement in neurologically intact survival in the epinephrine group, despite the higher rate of overall survival (8).

Given the uncertainty surrounding the efficacy of epinephrine in cardiac arrest, the PARAMEDIC2 trial is a welcome addition to the current body of literature. It is the first rigorous experimental study to confirm that epinephrine may actually have longer-term benefits beyond ROSC. This study showed a modest increase in 30-day survival associated with epinephrine - a finding that has never previously been demonstrated. However, it confirmed what prior observational studies have suggested: that epinephrine may actually worsen neurologic outcomes among cardiac arrest survivors. Given the immense psychological, spiritual, and economic costs associated with prolonging life in the absence of neurologic recovery, this study compels clinicians to radically rethink the risks and benefits of epinephrine in cardiac arrest. It is clear from the results of this trial that the routine administration of epinephrine in all cases of cardiac arrest is not warranted.

The physiologic explanation for the PARAMEDIC2 findings is not clear. The authors postulate that, despite improving macrovascular cerebral perfusion pressures, epinephrine may cause microvascular ischemia in the brain, thereby worsening anoxic brain injury. This theory is supported by animal studies in which this exact phenomenon was observed (9). They also suggest that the brain may have less capacity for recovery from anoxia compared to other organs. In addition to its effects on the brain, epinephrine has also been shown to worsen postresuscitation myocardial depression in an animal model, an effect which would be expected to worsen cerebral perfusion (10). Whatever the cause, the deleterious effects of epinephrine on neurologic outcomes should give clinicians pause when considering its use in adult cardiac arrest patients.

Before giving up on epinephrine entirely, however, the resuscitation community should seek to identify subgroups in which it may yield benefit. The PARMEDIC2 trial included all nonpregnant adult patients with out-of-hospital arrest, with the exception of those associated with asthma and anaphylaxis, for which epinephrine would be expected to offer clear benefit. There was no subgroup analysis examining the timing of epinephrine dosing relative to the onset of arrest. However, observational studies have suggested benefit for early administration of epinephrine compared to later administration $(8,9)$. Additionally, in the study examining patients with ROSC (discussed above), delayed administration of epinephrine was associated with worse outcomes (7). These findings reinforce the AHA recommendation to give epinephrine as early as possible in non-shockable cardiac arrest, but do not define a window in which epinephrine clearly benefits the patient. It is possible that epinephrine may offer benefits when given early, particularly in cases of witnessed arrest with bystander CPR and early defibrillation.

It is also possible that alternate dosing regimens for epinephrine may yield different outcomes. The PARAMEDIC2 trial used the standard AHA recommendation of intermittent $1 \mathrm{mg}$ bolus doses. It is clear that higher doses 
are not beneficial and worsen neurologic outcomes (7). Lowerdose epinephrine has been shown to produce comparable survival rates and neurologic outcomes to standard-dose epinephrine (11). But again may yield benefit in certain subgroups based on timing of administration. It is certainly reasonable to postulate that lower-dose regimens may mitigate some of the harm associated with epinephrine use.

While further studies of epinephrine are ongoing, it would be helpful for the AHA to review its guidelines regarding the use of epinephrine. Before the PARAMEDIC2 trial, the largest placebo-controlled study of epinephrine suffered from under-recruitment related to public outcry against deviation from "standard of care." (12). By softening language around recommendations, the AHA can free investigators from this unproven "standard of care," thereby enabling proper scientific scrutiny of the role of epinephrine in cardiac arrest. This would also enable clinicians to withhold epinephrine in cases where it is unlikely to benefit the patient, potentially preventing the tragedy of survival in the absence of neurologic recovery. However, until guidelines are changed, clinicians should continue to follow AHA protocols given their widespread acceptance by the medical community and the need for expert guidance in this important area of medicine.

\section{Acknowledgements}

None.

\section{Footnote}

Conflicts of Interest: The authors have no conflicts of interest to declare.

\section{References}

1. Nolan JP, Hazinski MF, Billi JE, et al. Part 1: Executive summary. Resuscitation 2010;81 Suppl 1:e1-25.

Cite this article as: Jung J, Rice J, Bord S. Rethinking the role of epinephrine in cardiac arrest: the PARAMEDIC2 trial. Ann Transl Med 2018;6(Suppl 2):S129. doi: 10.21037/atm.2018.12.31
2. Long B, Koyfman A. Emergency Medicine Myths: Epinephrine in Cardiac Arrest. J Emerg Med 2017;52:809-14.

3. Olasveengen TM, Sunde K, Brunborg C, et al. Intravenous Drug Administration During Out-of-Hospital Cardiac Arrest: A Randomized Trial. JAMA 2009;302:2222-9.

4. Olasveengen TM, Wik L, Sunde K, et al. Outcome when adrenaline (epinephrine) was actually given vs. not given - post hoc analysis of a randomized clinical trial. Resuscitation 2012;83:327-32.

5. Hagihara A, Hasegawa M, Abe T, et al. Prehospital Epinephrine Use and Survival Among Patients With Outof-Hospital Cardiac Arrest. JAMA 2012;307:1161-8.

6. Dumas F, Bougouin W, Geri G, et al. Is Epinephrine During Cardiac Arrest Associated With Worse Outcomes in Resuscitated Patients? J Am Coll Cardiol 2014;64:2360-7.

7. Lin S, Callaway CW, Shah, Prakesh S, et al. Adrenaline for out-of-hospital cardiac arrest resuscitation: A systematic review and meta-analysis of randomized controlled trials. Resuscitation 2014;85:732-40.

8. Perkins GD, Ji C, Deakin CD, et al. A Randomized Trial of Epinephrine in Out-of-Hospital Cardiac Arrest. N Engl J Med 2018;379:711-21.

9. Ristagno G, Sun S, Tang W, et al. Effects of epinephrine and vasopressin on cerebral microcirculatory flows during and after cardiopulmonary resuscitation. Crit Care Med 2007;35:2145-9.

10. Angelos MG, Butke RL, Panchal AR, et al. Cardiovascular response to epinephrine varies with increasing duration of cardiac arrest. Resuscitation 2008;77:101-10.

11. Fisk CA, Olsufka M, Yin L, et al. Lower-dose epinephrine administration and out-of-hospital cardiac arrest outcomes. Resuscitation 2018;124:43-8.

12. Jacobs IG, Finn JC, Jelinek GA, et al. Effect of adrenaline on survival in out-of-hospital cardiac arrest: A randomised double-blind placebo-controlled trial. Resuscitation 2011;82:1138-43. 